\title{
An Instructional Framework Supporting Personalized Learning on the Web
}

\author{
Kyparisia A. Papanikolaou \\ Research Fellow \\ Department of Informatics and \\ Telecommunications, University of Athens \\ spap@di.uoa.gr
}

\author{
Maria Grigoriadou \\ Associate Professor \\ Department of Informatics and \\ Telecommunications, University of Athens \\ gregor@di.uoa.gr
}

\begin{abstract}
In this paper an instructional framework is proposed for supporting personalised learning in the context of webbased adaptive educational hypermedia systems. A learning-focused approach of instruction is adopted aiming to address learners' needs and accommodate their learning style preferences and progress during interaction. In particular, the proposed framework unifies several processes that form system's adaptive behaviour such as the lesson generation process (guidelines for planning the content and delivery of instruction), the development of the domain model and the individualized navigation and study support provided. The way this framework has been implemented in a web-based adaptive educational hypermedia system is also presented. Finally, an empirical study has been conducted to investigate learners' attitudes towards the proposed framework and the first results are described.
\end{abstract}

\section{Introduction}

Adaptive Educational Hypermedia (AEH) systems incorporate the idea of supporting personalized learning. In this context, it is important to consider the educational potential of adaptation and investigate the educational implications of the use of adaptive educational environments over the Internet.

Specifically, personalization in an educational context needs a certain understanding of the learner as well as of the tasks that are important to learning. Thus, it is necessary to determine which instructional approach is suitable for learners of a particular profile and how this approach could be supported by the knowledge representation scheme of a Web-based environment. To this end, theories from the areas of Instructional Design, Adult Learning and Learning Styles are valuable resources.

In this paper we propose an instructional framework which models the adaptive behaviour of an AEH system by providing guidelines for planning the content, delivery and presentation of educational content to each individual learner. The way this framework has been integrated in a recently developed AEH system, named INSPIRE (INtelligent System for Personalized Instruction in a Remote Environment) is presented.

\section{The Instructional Framework}

Different pedagogical approaches have been adopted in AEH systems. In several cases, these approaches are based on a theoretical background that formulates the central concept of the interactions that take place between the system and the learner (Henze et al., 1999) and in other cases they simulate teachers' expertise formulating specific teaching strategies which are included in the system and guiding the development of the educational content (Specht et al., 1998).

In the proposed framework the synergy of two instructional design theories, Elaboration Theory [4] and Component Display Theory [6], with the learning style theory, [7], is adopted with the aim to build a "learningfocused" approach of instruction and support learners construct their own knowledge. Learners are provided with a variety of resources - such as authentic activities, theory, examples, collaborative tasks - which are proposed in a sequence that matches learners' knowledge level and preferred way of studying. Furthermore, the proposed framework formulates guidelines for structuring the domain knowledge and developing the appropriate educational content. This is an important issue for the development of AEH systems where the domain knowledge model is incorporated in the system and it mainly influences the effectiveness of adaptation.

Different processes that are modelled in the particular framework concern both the content and delivery of individualized instruction. Planning the content of instruction concerns the selection of the appropriate concepts on which learners should focus according to their knowledge level, whilst planning the delivery of instruction deals with the appropriate selection and presentation of educational material for the particular concepts following learners' knowledge level and 
learning style. The above processes build on the domain knowledge of the system aiming to gradually present it to learners.

In more detail, building elements of the Elaboration Theory (ET) that have been considered in planning the content of instruction are: selection of the fundamental concepts for each specific learning goal, sequencing of these concepts in the lessons contents, synthesizing of the interrelations among the domain concepts and summarizing instructional content. The aim is to organize instruction in order to show interrelationships among the different concepts of the domain following specific strategies [8]. The Component Display Theory (CDT) has been mainly exploited for the development of educational material for the main domain concepts tailored to specific performance levels which learners should achieve in order to cover the concepts. Moreover, in our approach, aiming to build a "learning focused" framework, the educational material on each particular level of performance is enriched by multiple types of resources such as authentic and meaningful activities and collaborative tasks.

Lastly, the learning style model of Honey and Mumford [7], which suggests four types of learners: Activists, Pragmatists, Reflectors, and Theorists, has been adopted as the basis of determining the presentation of the educational material on each of the performance levels. The particular model of learning styles focuses on adults' preferences of specific types of activities and instructional material, and provides useful guidance for associating the sequencing of type-specific instructional material with learners' preferences and studying attitudes in order to achieve certain learning outcomes.

\section{Personalized Lesson Generation}

INSPIRE [5], is a prototype AEH system designed to support Web-based instruction as well as traditional classroom-based teaching as a supplementary resource. Based on learners' learning goals, INSPIRE plans a sequence of lessons that accommodate learners' knowledge level, progress and learning style aiming to support learners to gradually achieve each of their goals. With regards to the adaptive dimension of INSPIRE, the selection of the lessons contents (the curriculum sequencing technology [1] is adopted), and the provided support over learners' navigation and study (the adaptive navigation support and adaptive presentation technologies [1] are adopted) are both based on the proposed instructional framework. However, learners are not restricted to follow system suggestions, as they are always able to navigate through the hyperspace or to intervene in the adaptive behaviour of the system by modifying their model (the learner model that INSPIRE stores and updates for each learner) or by deactivating system's adaptive behaviour.

\subsection{Planning Content and Delivery of Instruction}

In INSPIRE, learners have always the option to select and study the learning goal they prefer independently of their previous selections; all the material necessary for their study is provided when a learning goal is selected. In particular, INSPIRE plans the content of instruction for the selected learning goal, i.e. selects the contents of a sequence of lessons that gradually support learners to achieve their goal. These lessons are organized around specific outcome concepts of the particular learning goal. Thus, each lesson includes a learning goal, outcome concepts (followed by their prerequisites and related ones) and educational material associated with each of the outcome concepts and their prerequisite ones. Figure 1 shows the contents of a lesson (Navigation Area) for a learner who selected the learning goal "Describe the role of cache memory and its basic operations" or briefly "Cache Memory". Thus, the particular lesson includes two outcome concepts, "The role of cache memory" and "Mapping techniques". Once the learner selects an outcome concept to study, i.e. clicks on the corresponding concept name in the Navigation Area, a list of links to educational material pages appears below the concept name. The educational material of each outcome concept is organized in three levels, i.e. Remember, Use, and Find (represented by specific icons), which correspond to the three levels of performance proposed by CDT.

The complete conceptual structure provided for each learning goal includes concepts that should be taught according to ET prescriptions (selection). These concepts have different degree of importance for the accomplishment of a goal: outcome, prerequisite and related concepts. In this way, interrelations among the different concepts of a learning goal are outlined (ET: sequencing, synthesis). In particular, the concepts that are fundamental to the accomplishment of a goal are named outcome concepts. In order to fulfil a learning goal, learners should study all the outcome concepts and be successfully assessed to all of them. Prerequisite and related concepts are associated with each outcome concept complementing its presentation. Prerequisite concepts are essential in order learners to study the outcome concept, and related concepts are primitive concepts used in the educational material of the outcome. Note that in the proposed structure, the concepts are independent elements that can be reused in different learning goals. The educational material pages of prerequisites and outcomes, at each particular level of performance, are used as learning objects, and their representation follows the ARIADNE recommendation [2].

The outcome concepts of a learning goal are presented gradually (curriculum sequencing technology) following a "layered structure" which is in accordance with the ET prescriptions for sequencing: at the first layer the simplest 
and more fundamental concepts are proposed to learners, providing an overview of the learning goal, and then, subsequent layers add complexity or detail to a part or aspect of the learning goal. In Table I the layered structure of the outcome concepts is presented in the column labelled "Layer".

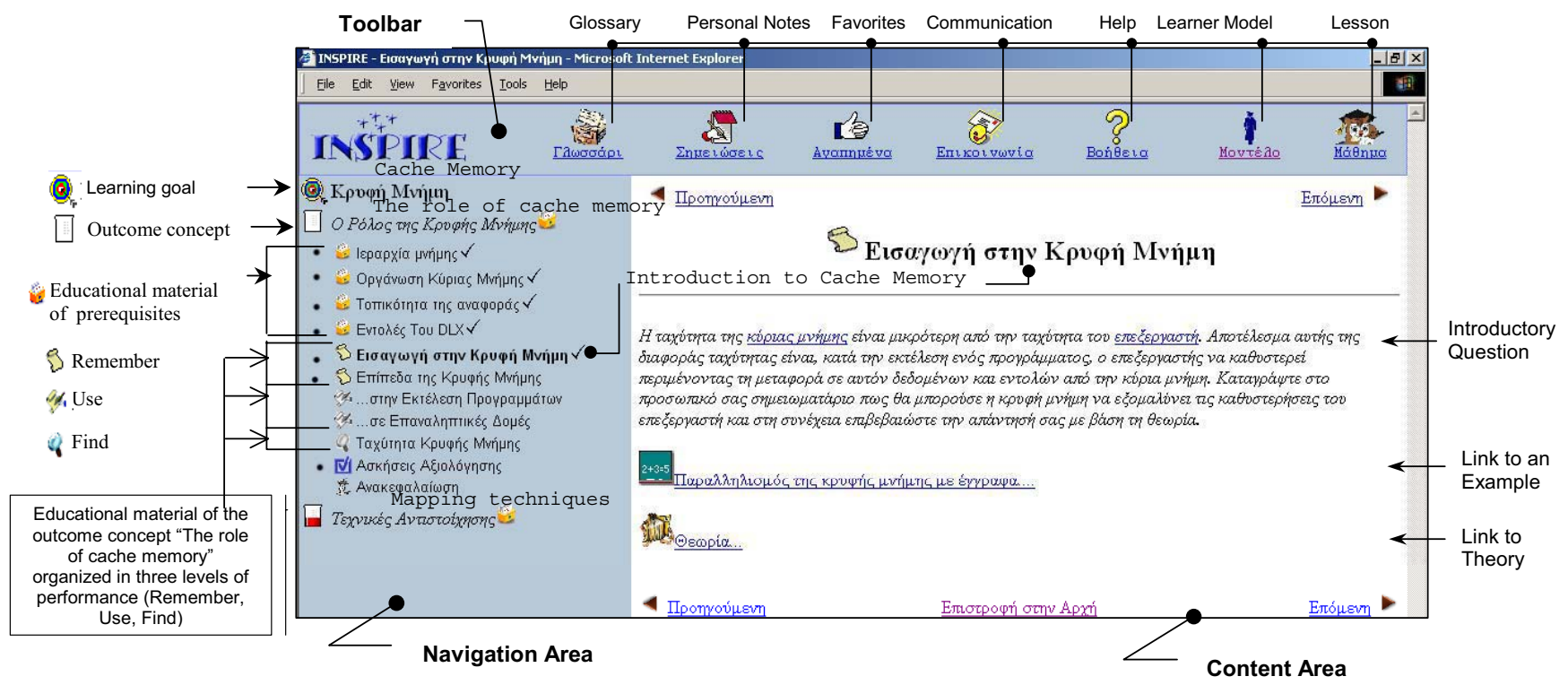

Figure 1. Presentation of the educational material page entitled "Introduction to Cache Memory" for an Activist. Note that colored icons of educational material pages in the Navigation Area are marked with a bullet for clarity

Thus, in the beginning of the interaction (when the learner first $\log$ on the system), the outcomes of the first layer appear to the learner, as in Figure 1. Afterwards, the layer of the outcome concepts proposed to the learner is determined by the system based on learner's progress, i.e. in order to go on to the next layer the learner should be "Advanced" on the outcome concepts of the current layer. Additionally, the concepts of each subsequent layer, starting from the first layer, enrich those of the previous ones, augmenting the domain presented to learner.

INSPIRE also plans the delivery of instruction which deals with the selection of the educational material that accompany each outcome concept included in the lesson contents. The educational material developed for each outcome is structured following the three levels of performance proposed by CDT (see Figure 1 - Navigation Area: Educational material of the outcome concept). Actually, one or more educational material pages are developed for each level of performance. Moreover, following the prescriptions of the CDT according to which each level of performance should include presentation, practice and test items, each page of educational material is comprised of a variety of knowledge modules depending on the level of performance of the page:

- Pages of the Remember level of performance include knowledge modules that introduce the concept and make learners speculate on newly introduced ideas, such as theory presentations, questions, and instances of the concept (see Figure 1, Content Area).

- Pages of the Use level of performance include knowledge modules that support learners to apply the concept to specific case(s), such as hints from theory, application examples, exercises, activities using computer simulation, exploratory activities.

- Pages of the Find level of performance include knowledge modules that stimulate learners to find a new generality, principle, procedure, such as activities using computer simulation, exploratory activities, and case studies. At this level, the learner submits an essay to the tutor and collaborative work is proposed.

In addition, the educational material of each outcome, following the guidelines for developing content for distance learning settings, includes: (a) an introductory page that introduces the concept and presents the learning outcomes posed; (b) a summary that reviews the content presented for the particular concept;(c) an assessment test.

With regards to the prerequisite concepts of each outcome, brief descriptions are presented on a page of educational material, whilst related concepts are associated with definitions included in the glossary of the system.

Although, all the above material is available to learners for the concepts included in the lesson contents, the educational material pages that are proposed to him/her for study (adaptive navigation support technology) are determined, and accordingly annotated, based on specific instructional strategies that take into 
account the type of each concept (outcome or prerequisite) and the knowledge level of the learner on the particular concept. For example, in case that the learner is a novice on an outcome concept, i.e. his/her knowledge level is characterized as "Inadequate", then the pages of the Remember level for the particular concept and all its prerequisites will be proposed. Following learner's progress, the system proposes the pages of the Use level (when his/her knowledge level becomes "Almost Adequate") and finally of the Find level (when his/her knowledge level becomes "Adequate") (see [5] for more details). This way, we provide individual navigation advice following learners' progress, without restricting the educational material and limiting learners' freedom to browsing.

In particular, system's suggestions on the concepts and educational material that the learner is ready to study are used to annotate the corresponding links of lessons contents in the Navigation area. For example, in Figure 1, learner's knowledge level on the outcome concept "The role of cache memory" is characterized as "Inadequate" and thus, the educational material pages of the Remember level of performance for the particular outcome and its prerequisites are proposed to the learner (marked with colored icons instead of black and white icons that appear next to the rest of the links).

Table I. The conceptual structure of the learning goal "Describe the role of cache memory and its basic operations".

\begin{tabular}{|c|c|l|l|l|}
\hline No & Layer & \multicolumn{1}{|c|}{ Outcome Concepts } & \multicolumn{1}{|c|}{ Prerequisite Concepts } & \multicolumn{1}{|c|}{ Related Concepts } \\
\hline 1 & 1 & $\begin{array}{l}\text { The Role of Cache } \\
\text { Memory }\end{array}$ & $\begin{array}{l}\text { Memory hierarchy, Main memory } \\
\text { organization, Locality of reference, DLX } \\
\text { instructions }\end{array}$ & $\begin{array}{l}\text { Sequential program execution, CPU, } \\
\text { Main memory, Looping constructors }\end{array}$ \\
\hline 2 & 1 & Mapping Techniques & Main memory organization & $\begin{array}{l}\text { Modulo operation, Block, Memory } \\
\text { capacity }\end{array}$ \\
\hline 3 & 2 & Basic Cache Operations & Main memory organization & Byte-addressable memory, Block \\
\hline 4 & 2 & Identification & Digital circuits, CPU Read/Write operation & $\begin{array}{l}\text { Bus organization, Comparator, } \\
\text { Multiplexer, Logic gates }\end{array}$ \\
\hline 5 & 2 & Placement Operation & Digital circuits, CPU Read/Write operation & Bus organization, Multiplexer \\
\hline 6 & 2 & Replacement Operation & Digital circuits, CPU Read/Write operation & Bus organization, Multiplexer \\
\hline 7 & 2 & Write Operation & Digital circuits, CPU Write operation & Bus organization, Buffer \\
\hline 8 & 3 & Cache Performance (CP) & CPU performance, Memory performance & Access time, Clock cycles per instr \\
\hline
\end{tabular}

\subsection{Planning the Presentation of the Content}

In INSPIRE learner's learning style is automatically recognized through the submission of the appropriate questionnaire (Honey \& Mumford, 1992) or it is declared by the learner. According to the proposed instructional framework, in the generated lessons of INSPIRE, the same educational material modules are reused following different instructional strategies that focus on different perspectives of the presented topic depending on the learning style of the learner. The implementation of these strategies results to changing the order and mode (embedded or appear as link) of presentation of the modules in an educational material page (adaptive presentation technology). For example, in Figure 1, the Content Area presents an educational material page of the Remember level of performance as it appears to an Activist. The presentation of the educational material to Activists, who are more motivated by experimentation and attracted to challenging tasks, focuses on activities. Thus, inquisitory presentation of theory is adopted, which provides initially a question, embedded at the top of the educational material page, aiming to motivate learners to use their prior knowledge, and then provides appropriate examples and theory. In case of a Reflector who tend to collect and analyze data before taking action, theory would appear embedded at the top of the page and then the example and a self-assessment question would be provided.

This approach aims to enhance learning by matching the dominant learning preferences of learners with the appropriate sequencing and presentation of educational material. In addition it stimulates learning style growth and collaboration by providing learners with multiple types of educational material resources to study and work with. Lastly, this way we face the problem of rewriting the same content for each learning style category; a commonly used approach for the development of instructional material tailored to different learning style categories [3].

\section{Empirical Study}

In the frame of the formative evaluation of INSPIRE we investigated learners' subjective estimations of the support provided by the system throughout their study by means of the adopted instructional framework. Two subsequent experiments were conducted in which 33 students were involved. During the experiments participants studied for two hours the learning goal "Cache memory" following a specific usage scenario and answered to several questions about different aspects of the system (a sample is presented in Table II). As far as the instructional framework is concerned, participants 
were asked to state their opinion about the usefulness of the planning the content and delivery processes.

Learners commented positively on the structure of the content and they noted that especially the adopted conceptual structure (outcome, prerequisites, and related concepts) provides a general overview of the subject matter in an easy to follow way and a comprehensive presentation of the subject that includes all the appropriate material covering possible gaps in their prior knowledge (see Table II, Question 1). Also, they commented that the provided educational material (because of its type, variety and structure) is easier to study, to understand, and find specific information within it compared to the handouts of the module. Participants positively commented on the structure of the educational material of the outcome concepts, i.e. organized in three levels of performance (see Table II, Question 2). In particular, they noted that combining theory with application in a gradual way results in a simple and simultaneously comprehensive presentation of the fundamental concepts of the domain. Moreover, two of the participants commented that this way resembles the way people approach new concepts. Also, participants reported that the usage of multiple types of educational material "kept them concentrated" and provided them with the opportunity to "gradually approach the main concepts of the goal through various perspectives, satisfying learners with different studying attitudes" (see Table II, Question 3).

Table II. A sample of the questions posed to students

Question 1: Do you think that the concepts' organization in outcome concepts followed by their prerequisites and related facilitated your study?

Question 2: Does the structure of the material on different levels of performance (Navigation Area), Remember, Use, Find, support the accomplishment of the learning outcomes posed?

Question 3: Does the multiple knowledge modules on each outcome concept (theory, examples, activities, etc.) support the understanding and use of the concept?

Question 4: Does the presentation of multiple knowledge modules on each concept (theory, examples, activities, etc.) through links (Content Area) facilitate your study?

Question 5: Do you think that providing gradually the main concepts of the learning goal facilitates your orientation in the lesson contents and supports knowledge assimilation?

Also, students found useful the presentation of multiple types of knowledge modules in each educational material page in the Content Area (see Table II, Question 4). In particular, they commented that although studying in front of a computer screen for an extended period of time is very fatiguing, the idea of structuring a page in multiple areas is helpful. Actually it facilitates study and at the same time, this way the system supports them in efficiently organizing their study and provides learners with the initiative to select the educational material to study next.

Lastly, with regards to the gradual presentation of the concepts of a learning goal (see Table II, Question 5), this approach seems to satisfy most of the participants, especially in cases where they are not familiar with the subject. However, one participant commented that this approach prevents him from formulating an overview of the subject matter.

\section{Conclusions}

In INSPIRE, instructional design theories are combined with the learning styles theory aiming to develop an adaptation framework that is educationally effective and technologically feasible. This framework unifies several processes that mainly affect system's adaptation guiding the design and development of AEH systems, such as structuring the domain model and developing the educational material, planning the content, delivery and presentation of educational material, and providing the appropriate navigation and study support to learners.

\section{References}

[1] P. Brusilovsky, "Methods and Techniques of Adaptive Hypermedia", In: P.Brusilovsky, A.Kobsa, and J.Vassileva (eds.), Adaptive Hypertext and Hypermedia, Netherlands: Kluwer Academic Publishers, 1998, 1-44.

[2] ARIADNE project. Available online at: http://ariadne.unil.ch/Metadata/ [02/2000].

[3] C. McLoughlin, "The implications of the research literature on learning styles for the design of instructional material". Australian Journal of Educational Technology 15 (3) 1999.

[4] C.M. Reigeluth, and F.S. Stein, "The elaboration theory of instruction". In: C.M.Reigeluth (ed.): Instructional design theories and models: An overview of their current status, Lawrence Elrbaum Associates, Hillsdale, NJ, 1983.

[5] K.A. Papanikolaou, M. Grigoriadou, G.D. Magoulas, and H. Kornilakis, "Towards New Forms of Knowledge Communication: the Adaptive Dimension of a Web-based Learning Environment", Computers and Education, 39 (4) (2002) 333-360

[6] M.D. Merrill, "Component Display Theory". In: C.M.Reigeluth (ed.): Instructional design theories and models: An overview of their current status. Hillsdale: Lawrence Erlbaum Associates, 1983.

[7] P. Honey and A. Mumford, "The manual of Learning Styles", Peter Honey, Maidenhead, Published and Distributed by Peter Honey, 1992.

[8] S. Hoffman, "Elaboration theory and hypermedia: Is there a link?", Educational Technology, 37(1) (1997) 57-64.

[9] M. Specht and R. Oppermann, "ACE - Adaptive Courseware Environment", The New Review of Hypermedia and Multimedia, 4 (1998) 141-161. 
[10] N. Henze, K. Naceur, W. Nejdl and M. Wolpers, "Adaptive Hyperbooks for constructivist teaching", Kunstliche Intelligenz, 4 (1999) 26-31. 